\title{
Cortex, striatum and cerebellum: control of serial order in a grooming sequence
}

\author{
Kent C. Berridge ${ }^{1}$ and Ian Q. Whishaw ${ }^{2}$ \\ ${ }^{1}$ Department of Psychology, University of Michigan, Neuroscience Building 1103 E. Huron St., Ann Arbor, MI 48104-1687, USA \\ ${ }^{2}$ Department of Psychology, University of Lethbridge, Lethbridge, Alberta, Canada
}

Received August 22, 1991 / Accepted March 3, 1992

\begin{abstract}
Summary. Rats emit grooming actions in sequences that follow characteristic patterns of serial order. One of these patterns, a syntactic chain, has a particularly stereotyped order that recurs spontaneously during grooming thousands of times more often than could occur by chance. Previous studies have shown that performance of this sequence is impaired by excitotoxin lesions of the corpus striatum. In this study we examined whether the striatum is unique in its importance to this behavioral sequence or whether control of the sequence instead depends equally upon the cortex and cerebellum. In two experiments, a fine-grained behavioral analysis compared the effects of striatal ablation to the effects of motor cortex ablation, ablation of the entire neocortex, or ablation of the cerebellum. Cortical and cerebellar aspiration produced mere temporary deficits in grooming sequences, which appeared to reflect a general factor that was nonsequential in nature. Only striatal damage produced a permanent sequential deficit in the coordination of this syntactic grooming chain. We conclude that the striatum has a unique role in the control of behavioral serial order. This striatal role may be related to a number of sequential disorders observed in human diseases involving the striatum.
\end{abstract}

Key words: Movement - Sequence - Basal ganglia Frontal cortex - Cerebellum - Rat

\section{Introduction}

Control of behavioral sequences is a major task for motor systems. Behavior flows as a cascade of actions, and each action must occur in proper sequence in order for behavior to be effective. How are constituent actions coordinated within the brain into organized sequential patterns? This was called the problem of "action syntax"

Correspondence to: K. Berridge by Lashley (1951), who argued that it was one of the most fundamental problems faced by neuroscience.

The study of brain mechanisms of action syntax is advanced through demonstrations that sequential aspects of behavior can be related to specific aspects of brain function. A common procedure for producing behavioral sequences that can be related to brain function is to train animals to perform instrumental responses in an arbitrary order or to ask humans to perform sequential patterns of movement. An alternative approach, which was employed here, is to rely upon the natural sequential patterns seen in natural or species-typical behavior. Not only does natural behavior provide a rich source of patterned behavioral sequences but, more important from the point of view of understanding the neural substrates of action syntax, natural patterns of behavior do not depend upon explicit training or instruction. Disruption of a learned behavioral sequence after a neural manipulation could be due either to a failure of action syntax or to a failure of sequential memory. Studies of natural sequential patterns of behavior, on the other hand, are less liable to confound learning and memory with action syntax, and are better able to isolate processes relevant to behavioral sequencing per se.

Grooming patterns by rodents provide excellent examples of rule-governed sequential behavior, which can be linked by neurobehavioral studies to particular brain systems (Whishaw et al. 1983). Understanding the neural sequencing of grooming has significance that goes beyond grooming itself. More generally, an understanding of how neural systems coordinate patterns of grooming might expose basic principles that are relevant to the sequencing of other behavioral processes. The neural sequencing of grooming might even have clinical implications: some aspects of the recurring stereotypies of human obsessive/compulsive disorders have been suggested to have a special evolutionary relation to natural grooming. Although speculative, these suggestion are intriguing. Human compulsions often focus with intense emotion upon aspects of personal hygiene, for example, and commonly involve the ritualization of self-cleaning sequences (Rapoport 1989). 


\section{"SYNTACTIC" GROOMING CHAIN}

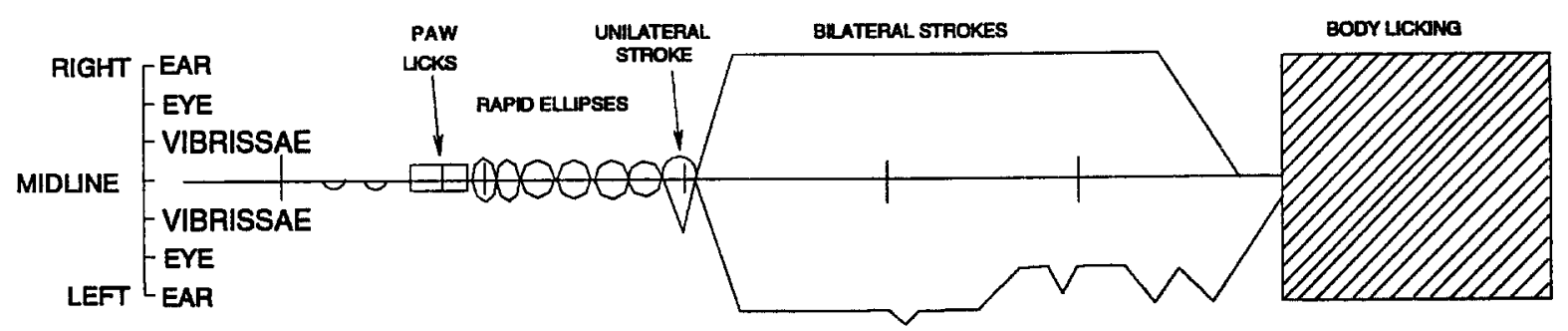

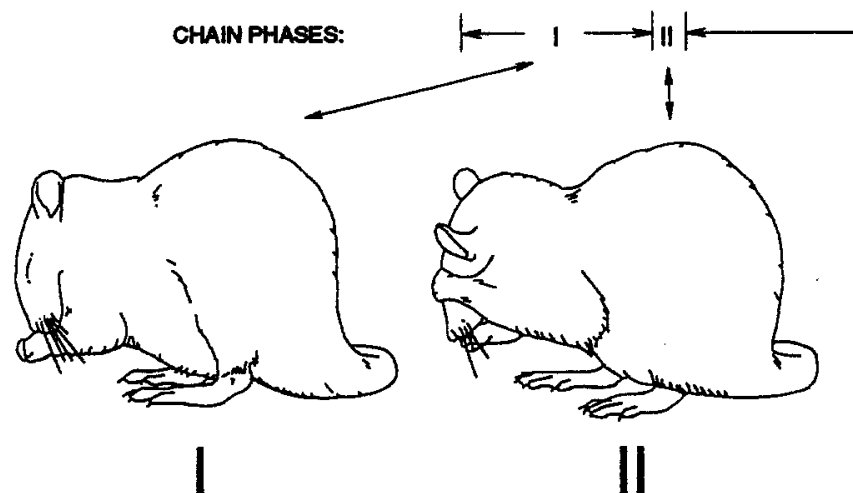

EUPTICAL STROKE
SMALL UNILATERAL STROKE
1

$-1$

0
Fig. 1. Choreographic transcription of an idealized syntactic grooming chain. Time proceeds from left to right. The horizontal axis represents the center of the nose. The line above the horizontal axis denotes movement of the right forepaw along the face; the line below the axis denotes movement of the left forepaw. Small rectangles denote paw licks. Large rectangle denotes body licks. Chain

Grooming actions by rodents are organized into sequences by a number of pattern-generating rules (Fentress and Stilwell 1973; Richmond and Sachs 1980; Ferron and Lefebvre 1982; Berridge 1990). Each species has its own characteristic pattern of grooming. These patterns are so reliable in structure that phylogenetic relationships can be inferred from the slight pattern differences between species nearly as well as from differences in physical traits (Berridge 1990). The most stereotyped sequential pattern is a syntactic chain of up to 25 grooming actions directed to the face and body, which are organized into 4 sequential phases in a fixed serial order (Fig. 1). Rodents of all major suborders (Myomorpha, Sciuromorpha, Hystricomorpha) show this syntactic chain with minor pattern variations. The syntactic chain pattern is emitted by rats during grooming at least 13000 times more often than could be expected by chance. Although syntactic chains form a relatively small proportion of total grooming, their extraordinary degree of sequential stereotypy makes them an excellent focus for studies of the brain substrates for behavioral sequencing. Outside of syntactic chains, sequential patterns of grooming exist but are more abstract, more difficult to predict, and less sensitive as measures to study neural manipulations (e.g., Fentress and Stilwell 1973; Richmond and Sachs 1980; Berridge 1990).

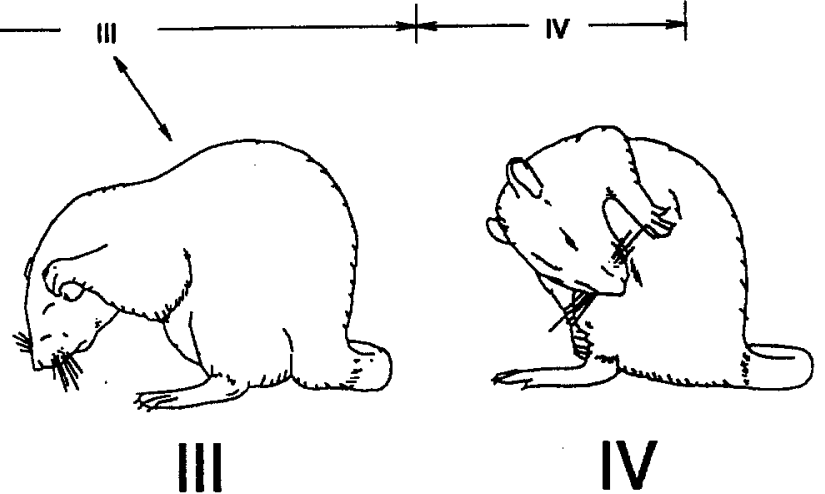

LARGE BILATERAL STROKE

BODY LCKING

\section{$\begin{array}{llll}2 & 3 & 4 & 5\end{array}$}

phases are: (I) 5 to 8 rapid elliptical strokes around the nose $(6.5 \mathrm{~Hz})$; (II) Unilateral strokes of small amplitude; (III) Symmetrically bilaterally strokes of large amplitude; (IV) Licking of the ventrolateral torso. Syntactic chains are typically completed (by Phase IV onset) within 5 seconds of the first Phase I ellipse (adapted from Aldridge et al. 1990)

The rule-governed pattern of syntactic chains appears to generated internally. The pattern is not disrupted by the loss of tactile sensory feedback from the face that is produced by trigeminal deafferentation (Berridge and Fentress 1987a). Decerebration studies have shown that this syntactic pattern is generated chiefly by the hindbrain. Even a metencephalic decerebrate preparation, which possesses only a medulla, pons, and cerebellum and which lacks a forebrain and midbrain, occasionally produces well-formed syntactic chains (Berridge 1989a). The normal stereotypy of the pattern, however, does require forebrain structures. Although decerebrates initiate this syntactic chain at a normal rate, they complete only half the normal percentage of chains that they begin. Chain completion is also halved by loss of dopaminergic projections to the neostriatum (Berridge 1989b) or by excitotoxic lesions of the striatum itself (Berridge and Fentress 1987b). A recent study using small lesions has shown that caudate-putamen damage need only occur bilaterally in the dorsolateral region of the anterior neostriatum in order to disrupt the completion of the sequence (Cromwell and Berridge 1990).

The equivalence of the sequential disruption produced by damage to the striatum, nigrostriatal projections, and pontine decerebration is striking (Berridge 1989b). Why are these deficits in syntactic grooming so similar? There 
are at least two alternative explanations for why the deficits might be equal. First, the equivalence of these deficits might indicate that striatal control of pontine structures is both necessary and sufficient for normal performance of the syntactic chain pattern. Because each of the manipulations removes striatal-related circuits from control of motor outputs, it might be that this loss alone is responsible for disruption of syntactic completion. A second possibility, less interesting from the standpoint of identifying the specific neural substrates of sequential control, is that the serial control of syntactic chains is such a vulnerable process that any forebrain lesion would reduce syntactic performance to decerebrate-like levels. Since the effects of other brain lesions on syntactic grooming chains have not been examined, this possibility cannot yet be ruled out. In order to choose between these two possibilities, it would be necessary to know whether damage to all major brain systems involved in movement execution would also impair syntactic chaining. In particular, it would be necessary to know whether similar deficits are caused by damage to structures such as the primary motor cortex, which has been linked to movement initiation and fine coordination, the supplementary motor cortex, which has been linked to movement planning and timing (e.g., Barone and Joseph 1989; Evarts and Wise 1984; Iversen and Mishkin 1970; Kurata and Wise 1988; Passingham et al. 1989; Zilles 1990), or the cerebellum, which has been linked to movement programming and to sequential alternation (e.g., Buchtel 1970; Dichgans 1984; Kirk et al. 1982). Rats still groom after loss of the motor cortex, whole neocortex, cerebellum, or striatum, although they have a variety of motor deficits (e.g., Whishaw 1990). Would damage to any of these brain structures impair the syntactic coordination of grooming chains in the same fashion?

The present study compared the effects of striatal and extra-striatal damage on the syntax of grooming. Experiment 1 of the present study compared the sequencing deficits produced by removal of the a) secondary motor cortex alone, b) primary and secondary motor cortex together, or c) entire neocortex, to the deficit produced by loss of d) the neostriatum in addition to these structures. In Experiment 2, the study was extended to the effect on syntactic grooming chains of bilateral ablation of the cerebellar cortex. Only striatal lesions produced an enduring disruption of grooming syntax. The results of these experiments supported the hypothesis that striatal systems play a unique role in the execution of this natural sequence.

\section{Experiments 1}

\section{Methods}

\section{Surgery}

Male Long-Evans rats (250-350 g) were anesthetized with sodium pentobarbital $(50 \mathrm{mg} / \mathrm{kg}$, ip) and atropine methyl nitrate $(5 \mathrm{mg} / \mathrm{kg}$, ip) for surgery. Lesions were made by visually-guided aspiration, using a dissecting microscope as in Whishaw, Nonneman and Kolb
(1981). The overlying skull was removed according to coordinates similar to Zilles (1990), and the meninges were cut and retracted. The area of cortex to be lesioned was demarcated by a $1 \mathrm{~mm}$ deep cut along the outer boundary, and the cortex within this boundary was aspirated. Four bilateral lesion groups ( 8 rats each in Motor Cortex lesion groups; 7 rats each in Decorticate and Destriate/ decorticate groups) and a surgical control group ( 8 rats) were used. Note: for the purposes of this paper, Primary motor cortex denotes all agranular frontal cortex, including Fr1, Fr3, and medial FL, in which low-intensity electrical stimulation produces forelimb movement. Supplementary motor cortex denotes agranular frontal cortex, essentially Fr2, in which stimulation does not induce movement (Donoghue \& Wise 1982; Neafsey et al. 1986; Neafsey 1990).

Lesion areas were: 1) Combined Primary and Supplementary Motor Cortex, which was bounded bilaterally by $1 \mathrm{~mm}$ to $5 \mathrm{~mm}$ lateral to the midline and from $-1 \mathrm{~mm}$ to $+5 \mathrm{~mm}$ anterior/posterior to bregma. 2) Supplementary Motor Cortex Alone, bounded bilaterally by $1 \mathrm{~mm}$ to $3 \mathrm{~mm}$ lateral to the midline and from $-1 \mathrm{~mm}$ to $+5 \mathrm{~mm}$ anterior to bregma. 3) Complete decortication, in which all neocortex and most cingulate cortex from dorsal midline to the ventral level of the rhinal fissure was removed along the entire longitudinal axis. 4) Striatum Ablation Combined with Decortication (Destriate/decorticate), in which the corpus callosum and neostriatum were removed by aspiration in addition to the neocortex. Bleeding was controlled with gelfoam. After hemostasis was achieved, the scalp was sutured. Bicillin ( $30000 \mathrm{U}$, im) was administered to prevent infection. (Note: Striatal aspiration without decortication was not performed in this study for two reasons. First, because selective striatal excitotoxin lesions have already been studied for their effects upon syntactic grooming chains (Berridge and Fentress 1987b; Cromwell and Berridge 1990). Second, because the aspiration lesion technique used in this study would destroy cortical fibers passing through the striatum, and so a selective striatal lesion is not possible by aspiration.)

\section{Maintenance}

Rats were housed individually on a $14: 10 \mathrm{~h}$ light: dark cycle, and were handled daily for at least 3 weeks prior to testing. Rats had free access for water and chow pellets. After striatal ablations, aphagic rats were nourished by 3 daily intragastric feedings of a liquid diet to maintain body weight (equal parts sweetened condensed milk and water, plus a vitamin supplement). Mcal intubation volume began at $6 \mathrm{ml}$, and ascended gradually over 2 days to $12 \mathrm{ml}$. Bedding was changed daily, and special nursing care was given as needed for destriate/decorticate rats. All cortical lesion groups began grooming, eating, and drinking within $48 \mathrm{~h}$, and quickly recovered body weight. Eating and drinking never recovered in destriate/decorticate rats, but body weight and hydration was maintained by intubation of the milk diet. Behavioral testing began 3 weeks after surgery.

\section{Behavioral testing}

Grooming sequences were elicited by spraying the fur with a light water mist. Grooming that is elicited in this way may not be identical in every respect to spontaneous grooming in the home cage (Fentress 1983; Whishaw 1990), but similar sequential patterns can be observed in both situations. After its fur was moistened, each rat was placed in a transparent test chamber, which was suspended over a mirror. The rat was allowed to groom for $30 \mathrm{~min}$, and its fur was moistened again every $10 \mathrm{~min}$. A ventral view of the head and rostral body was reflected by the mirror into the zoom lens of a videocamera, and a second camera captured a dorsolateral view, so that movement of the paws could be tracked accurately. Testing sessions were repeated daily until a total of at least 20 min of continuous grooming action had been videotaped for each rat. 


\section{Behavioral videoanalysis}

Two observers who were blind to the status of the rats scored each videotape in a slow motion analysis. Grooming behavior was analyzed for:

1. Overall frequency and duration. The grooming bouts of each rat were analyzed to determine the percentage of time spent in grooming and the average duration of grooming bouts (a bout was defined as a period of continuous grooming that contained no pause longer in duration than $5 \mathrm{~s}$ ).

2. Action type. At a finer level of analysis, the relative frequency of different grooming components was calculated for Forelimb strokes over the face by one or both paws, Paw Licking, in which the tongue was directed at one or both paws, and Body Licking, in which the tongue was directed at the torso or upper forelimb. Syntactic chains were identified by the criteria below, and each chain was transcribed frame-by-frame using a choreographic grooming notation system that continuously displays the position of the forepaws in relation to facial features (Berridge and Fentress 1986; Berridge 1990).

3. Efficacy of syntactic completion. Syntactic chains were analyzed for "goodness of completion". Phase I ellipses are small-amplitude forepaw strokes with elliptically shaped trajectories that are centered tightly at the tip of the nose, and which have a frequency of approximately $6 \mathrm{~Hz}$ (Fig. 1). Phase II strokes are small amplitude strokes that are nonrhythmic and usually unilateral or, if they are bilateral, are highly asymmetrical. Phase III strokes are large amplitude, symmetrical strokes made simultaneously with both paws. The beginning of a syntactic chain was defined as the occurrence of a full Phase I (a bout of five to nine consecutive bilateral "ellipses") followed immediately by one or more Phase II or Phase III strokes, with no intervening action of another type (Fig. 1). Grooming chains that were begun syntactically in this way were examined to determine the proportion that were completed syntactically to Phase IV (the initiation of body licking without interruption after Phase III) in order to assess sequencing capability.

4. Influence of ellipse timing and chain order upon syntactic completion. The probability that a syntactic chain will be completed through to Phase IV has been shown to be correlated with the rate at which elliptical strokes are generated during Phase I: faster ellipses predict a higher rate of syntactic chain completion (Berridge 1990). To discover whether this relationship can still be generated by a brain without a cortex or striatum, the cycle length of ellipses from complete and incomplete chains by rats with lesions was examined. In order to discover whether properties of syntactic chain organization remained constant over all chains emitted within a prolonged bout of grooming, the effect of chain order (initial chain of a video session versus later chains of the same session) on ellipse timing and chain completion also was examined.

5. Microstructure of syntactic chains. Finally, the fine structure of syntactic chains of rats from each lesion group was analyzed in terms of the symmetry and number of forelimb strokes contained within each chain, and the time to complete chains (by initiating Phase IV). The stroke-by-stroke microstructure of each chain was transcribed using a choreographic notation system for representing grooming sequences (Berridge and Fentress 1986; Berridge 1990).

Behavioral data were analyzed statistically using Analysis of Variance procedures (ANOVA) and Least Significant Difference post hoc tests (LSD).

\section{Histology}

At the completion of the experiment, rats were deeply anesthetized and perfused intracardially with a solution of $0.9 \% \mathrm{NaCl}$ and $10 \%$ formalin. The brains were removed, photographed, embedded, and placed in a $30 \%$ sucrose formalin solution. Each brain was frozen and sliced in 40 um coronal sections, mounted on slides, and stained with Cresyl violet. Maps of each lesion were made under magnification on a stereotaxic atlas (Paxinos and Watson 1989).

\section{Results}

\section{Histology}

Representative lesions and photomicrographs are depicted in Fig. 2. Lesions were small in the Supplementary Motor Cortex group. These lesions destroyed frontal cortex area $\mathrm{Fr} 2$ while sparing other frontal areas, parietal areas, and the cingulate areas, $\mathrm{Cg} 1, \mathrm{Cg} 2$, and $\mathrm{Cg} 3$. Lesions were larger in the Combined Primary and Supplementary Motor Cortex group: Fr1, Fr2, Fr2, Fr3, and FLf all were destroyed. Cingulate and parietal areas were spared. Decortication removed most of the cingulate cortex and virtually the entire frontal, parietal, and occipital neocortex above the rhinal fissure. Only ventral remnants of the cingulate cortex and small areas of neocortex adjacent to the rhinal fissure were spared in decorticates. The hippocampus, thalamus, and other subcortical areas were not damaged directly, although zones of calcification were found especially in the dorsal thalamus. Destriate/decorticate combinations additionally removed nearly all of the neostriatum (caudate/putamen). Portions of the ventral nucleus accumbens (ventral to anterior commissure) and the most ventroposterior remnants of the striatum were spared in destriate/decorticate rats. The hippocampus and thalamus were not damaged directly by striatal lesions but did show calcification.

\section{Overall grooming}

Control rats spent approximately $25 \%$ of the observation period in grooming. Rats that had motor cortex lesions were similar to control rats in the proportions of time spent in grooming. Rats that had lost their entire cortex or their cortex plus striatum spent a smaller proportion of time in grooming (decorticates $=14 \%$, LSD $p<0.05$; destriate/decorticate $=13 \%, p<0.01$, LSD) than the others (ANOVA $F(4,32)=6.15, p<0.01$ ). The proportion of time spent grooming by decorticates recovered to normal levels by the 10th day of observation, as described previously by Whishaw (1990), but the grooming of destriate/decorticate rats never recovered to normal proportions within the two month period of observation.

The duration of individual grooming bouts differed marginally between groups $(F(4,32)=4.8, p=0.07)$. A bout was defined as any continuous display of grooming that contained no pause of greater than $5 \mathrm{~s}$. The grooming bout duration of control rats averaged $26 \pm 7 \mathrm{~s}$. The grooming bouts of decorticate rats were slightly shorter than those of control rats (decorticates $=20 \pm 6 \mathrm{~s}$; LSD, $p<0.05$ ). The grooming bouts by rats with lesions only of the secondary motor cortex area were somewhat longer than controls $(39 \pm 12 \mathrm{sec}$; LSD, $p<0.05$ ). 


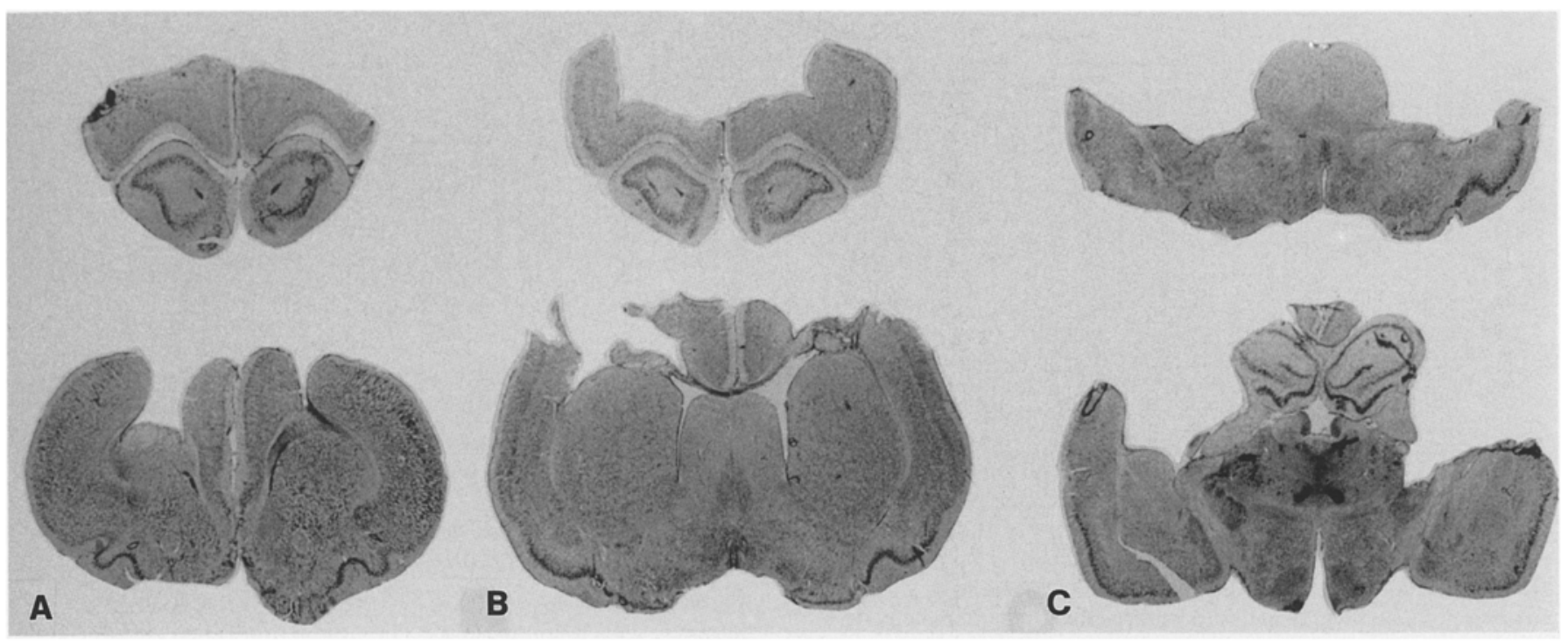

Control

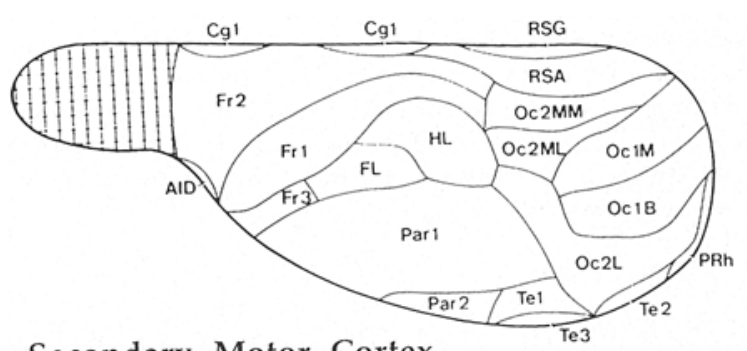

Secondary Motor Cortex

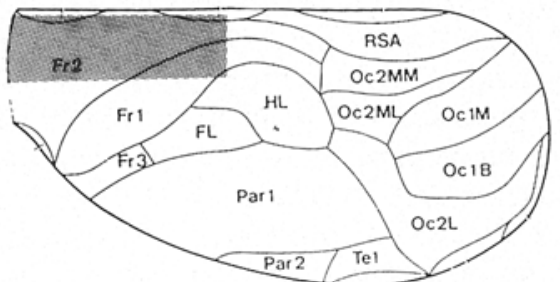

Motor Cortex

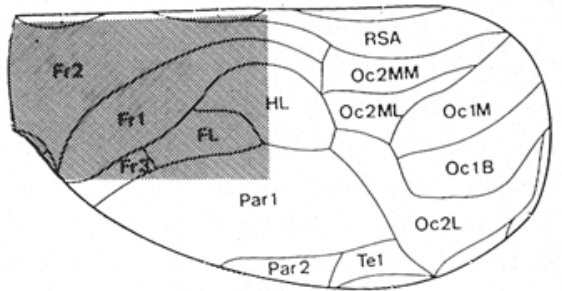

Decorticate

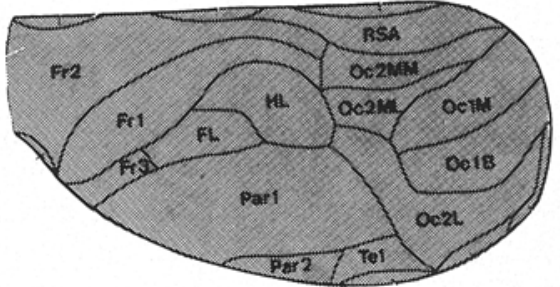

Fig. 2A-D. Experiment 1. Cortical and striatal lesions. Photograph of representative brains. A Supplementary motor cortex, B Primary and supplementary motor cortex, and $\mathbf{C}$ Destriate/decorticate. D Drawing shows areas of damage that were shared by rats whithin each group. Drawings are based on Zilles (1990)

\section{Syntactic chain completion}

During grooming, all groups initiated syntactic chains at similar rates per minute of grooming $(1.6 \pm 0.3$; ANOVA $F(4,32)=0.32, p>0.1)$. Chain initiation was defined as the sequential occurrence of Phase $I$ : at least 4 consecutive ellipses emitted at a rate of at least $5 \mathrm{~Hz}$, followed immediately by either Phase $I I$ : one or more unilateral medium amplitude strokes, and/or Phase III: one or more bilateral large amplitude strokes. A "syntactically complete" chain consists of Phases $I, I I, I I I$, and $I V$ arranged in that order (Fig. 1), and a number of such chains were observed in each session. Figure 5 shows representative examples for each lesion of syntactically completed chains and of chains that were initiated but left incomplete.

The percentage of chains that were completed syntactically to Phase IV (body licking) by control rats in this experiment was about $10 \%$ higher than in previous studies (e.g., Berridge 1989a; 1989b). This increase might have resulted from the different origin of the rats (LongEvans strain from the University of Lethbridge colony were used in this experiment whereas Sprague-Dawley rats from diverse suppliers were used in previous experiments) or from the prolonged habituation and longer, frequent test sessions used in this study (30 min observation vs $15 \mathrm{~min}$ used in earlier studies), which would have induced both longer grooming bouts and a higher incidence of completed chains (see below).

Cortical and striatal damage of all types produced detectable changes in the probability that an initiated chain would be completed (Fig. 3). The change was different for the different types of lesion. The deficit produced by cortical damage can be seen most clearly by examining chains emitted early in a grooming session (i.e., the first chain emitted by a rat during a session; Fig. 3). As a general rule, the initial chain of any session was less likely to be completed syntactically to Phase IV than were subsequent chains (i.e., a "warm up" or behavioral inertia phenomenon). Order of emission was a fac- 


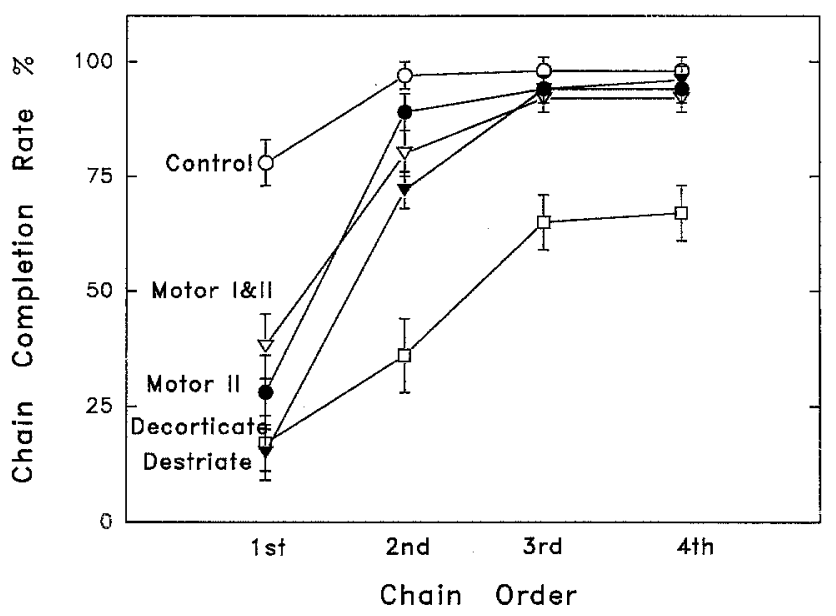

Fig. 3. Experiment 1. Syntactic chain completion: inertia within a grooming session. The percentage of first, second, third, and forth grooming chains begun within a session that were completed syntactically (using a stringent criterion for completion). For normal controls, the initial chain of any session was less likely to be completed than subsequent chains. Cortical damage enhanced this pattern. Striatal damage prevented recovery to normal levels later in a session

tor that influenced completion rate for all rats (ANOVA $F(1,32)=112.31, p<0.001)$. This was true even for intact rats: controls showed poorer completion rates for the initial chain of any session ( $78 \%$ completed to Phase IV) than for ' $n+1$ ' chains (i.e., all chains occurring after the first chain of the session) emitted later in the same session ( $99 \%$ complete; Newman-Keuls, $p<0.05)$. Intact control rats reached an asymptotic completion rate of $99 \%$ by the second chain of a session.

For rats with lesions, the "early chain" impairment of completion "recovered" more slowly, but completion reached an asymptotic level of recovery by the third chain of a session (Fig. 3). The magnitude of the early chain completion deficit was exaggerated by cortical damage (Fig. 3). Compared to the control rate of $78 \%$ completion, only $37 \%$ of 'initial chains' were completed syntactically by rats with primary motor cortex lesions; $30 \%$ were completed by rats with secondary motor cortex lesions; $16 \%$ were completed by full decorticates; and $17 \%$ were completed by rats that were destriate/decorticate.

Rats that had only cortical damage ascended nearly to control levels of completion for $n+1$ chains emitted later in a session (Fig. 3). If the striatum was damaged also, a more enduring impairment of syntactic completion was produced. Asymptotic rates of completion for destriate/decorticate rats never rose above $53 \%$ even for the last chains of a session. Destriate/decorticate completion rates for $\mathrm{n}+1$ chains remained below that of every other lesion group (Newman-Keuls, $p<0.01$ ).

Decorticate and destriate/decorticate rats also showed a further modification of syntactic chains that was unique to these groups. This was the occurrence of occasional imperfect Phase IV distortions of self licking (Fig. 4). After performing Phases $I, I I$, and $I I I$ in proper order, these rats would drop the head as if going to lick

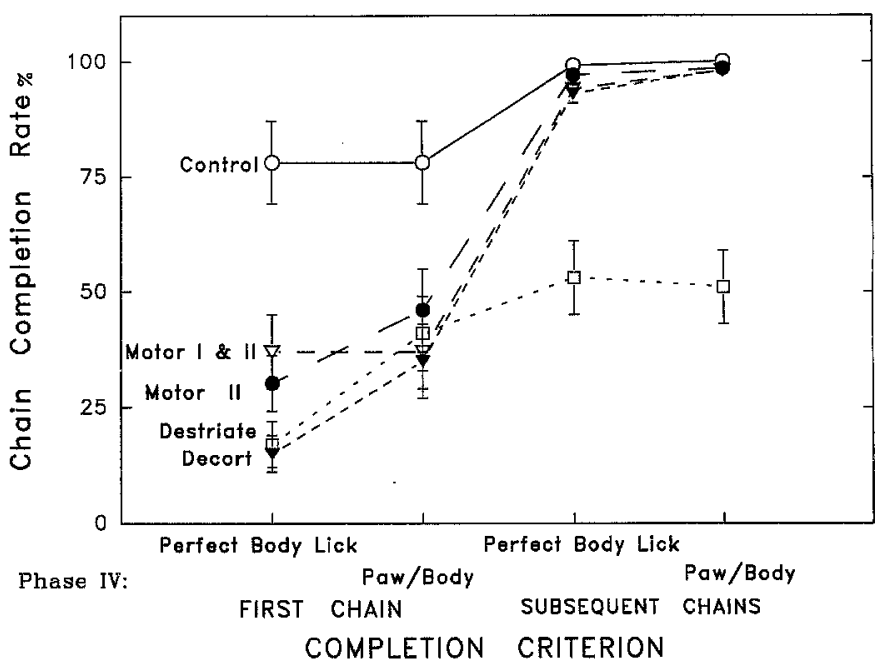

Fig. 4. Experiment 1. Changing the criterion for syntactic completion reveals lesion patterns. The completion deficits produced by cortical and striatal lesions are shown as the criterion for completion is gradually relaxed (left to right: Perfect phase $I V$ completion, by torso licking, of the first chain of a grooming session; Imperfect phase $I V$ completion, by forepaw licking, of the first chain of a session; Perfect completion of subsequent $n+1$ chains later in the session; Imperfect completion of later $n+1$ chains). By the most stringent criterion, even motor cortex lesions by themselves impair completion and decortication/striatal ablation produce a further impairment. By the least stringent criterion, only striatal ablation produces a permanent deficit

the ventrolateral torso as usual for Phase $I V$, but would stop midway and begin instead to lick the forelimb or shoulder ("imperfect" Phase IV). Normal rats complete chains by licking their ventral or lateral torso. If instances of "imperfect" body licking were included in rates of syntactic chain completion, then the completion rates of initial chains rose to $36 \%$ from $16 \%$ for decorticates, and to $41 \%$ from $17 \%$ for destriate/decorticate rats (decorticate and destriate/decorticate completion rates did not differ from each other for either type of initial chain). These rates were still below normal control levels. "Imperfect" instances of Phase IV were virtually never seen in the chains of normal or motor cortex rats, and were not often seen in ' $n+1$ ' chains even for decorticate or destriate/decorticate rats (Fig. 4).

These results can be summarized by a single theme. Chains can be completed succesfully with any lesion of the cortex or neostriatum, but the degree of perfection of the syntactic pattern is progressively degraded as the cortical lesion size grows, and is permanently impaired after striatal ablation. By the least stringent test of syntactic goodness, namely, Phase $I V$ completion of $n+1$ chains by licking either forelimb or body, only rats with striatal lesions were impaired. By a more difficult test of completion, that of at least imperfect completion of initial chains by either forelimb or body licking during Phase IV, any brain lesion reduces completion success below that of intact rats. Further, the reduction measured by this criterion was essentially the same for all cortex or striatal lesions. Finally, by the most difficult test of syntactic chain completion, that of perfect Phase 
Intact Control

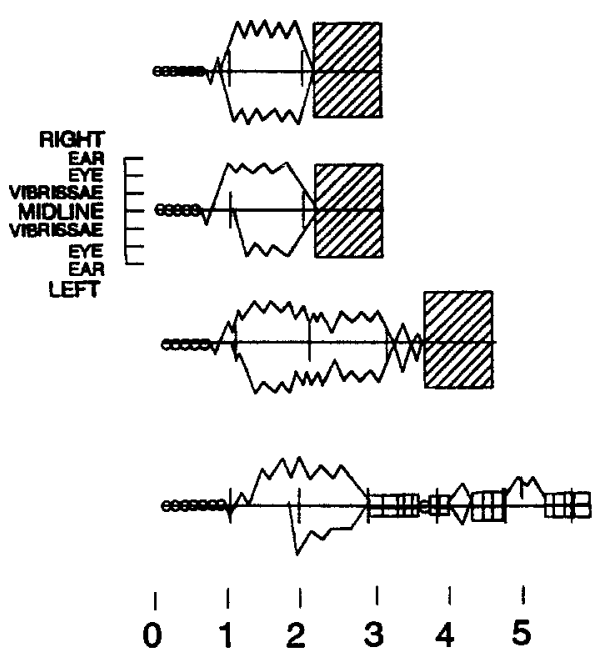

Motor I and II
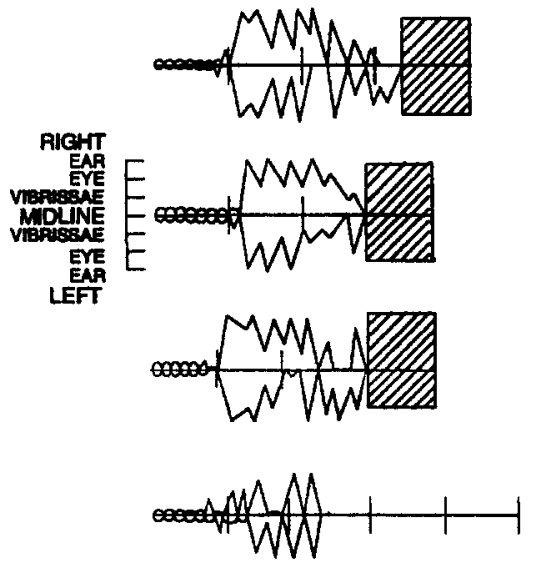

$\begin{array}{llllll}1 & 1 & 1 & 1 & 1 & 1 \\ 0 & 1 & 2 & 3 & 4 & 5\end{array}$
Motor II

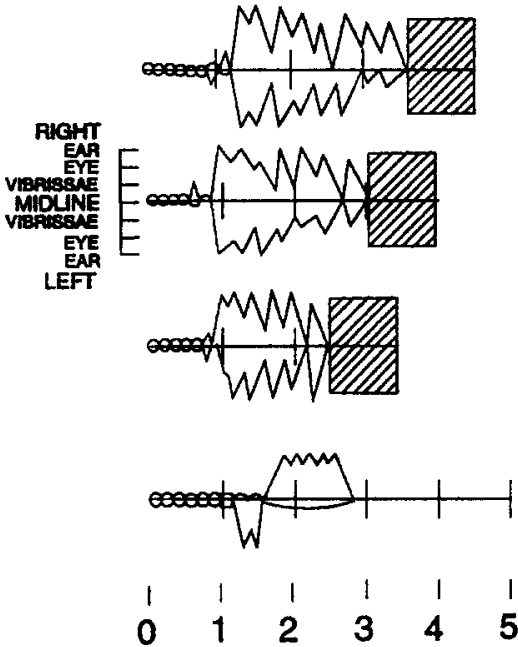

Decorticate
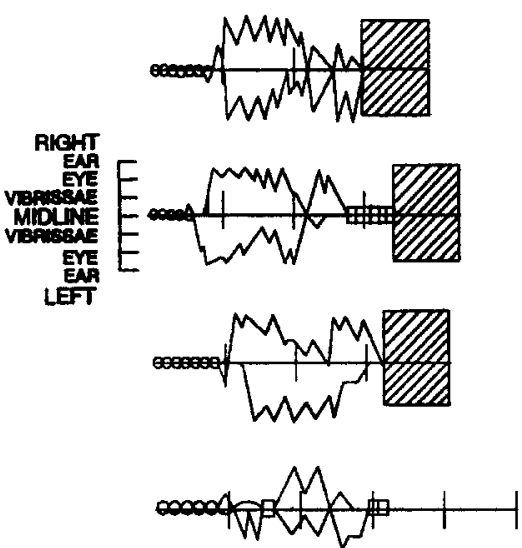

$\begin{array}{llllll}1 & 1 & 1 & 1 & 1 & 1\end{array}$
LEGEND

అO ELLPTKAL STROKES

$\checkmark$ LARGE BILATERAL STOKES

$A_{\rightarrow}$ SMAL UNLATERAL STROKES

EA PAW UCKS

BOOY LICKS

Fig. 5. Experiment 1. Notated syntactic chains. Actual samples from each group of 3 chains that were completed syntactically and of 1 chain that failed to be completed. Symbols are explained in the legend box. The position of the paws in relation to the face during each stroke is indicated by the stroke-amplitude marker near the second chain for each group. Time (in second) is shown at bottom

IV completion of initial chains by licking the ventral or lateral torso (not the forelimb), completion rates of full decorticate or destriate/decorticate groups dropped even further below normal, and also dropped below the motor cortex lesion groups, which in turn remained below control levels.

\section{Frequency of licking as a grooming component}

Do failures to complete syntactic chains result from an inability to engage in the motor component of Phase IV, namely, forepaw or body licking? The answer appears to be no. Control rats spent $50 \%$ of their grooming time licking their body (belly, side, or back) and $14 \%$ of their grooming time licking their paws and forelimbs. Rats with cortex lesions of all types spent proportions of grooming in body and forepaw licking (body $=46 \%$, forepaw $=20 \%$ ) that did not differ from control rats $(F(3,27)=0.65 ; p>0.1)$. Removal of the striatum reversed the relation between body and forepaw licking. Destriate/decorticate rats spent a smaller proportion of grooming time in body licking (20\%; ANOVA $F(4,32)=19.15 ; p<0.01 ;$ LSD $p<0.05$ compared to all other groups), but a greater proportion than normal of grooming time in licking their paws and forelimbs $(46 \%$; ANOVA $F(4,32)=8.69, p<0.01$; LSD $p<0.05$ compared to all other groups). This shows that it was not motor inability to perform licking that caused the deficits of syntactic chain completion. In fact, causation may 
proceed in the opposite direction. The reduced ability of destriate/decorticate rats to complete syntactic chains to Phase IV apparently "closed off" this particular route from face to body licking, and left these rats less likely to lick their bodies. Destriate/decorticate rats compensated by increasing the frequency of paw and forelimb licking as a grooming component. The increase of forepaw licking was fully equal to the decrease of body licking. Finally, it is important to note that destriate/ decorticate rats continued to have reduced syntactic completion rates even under our relaxed criterion of "imperfect" Phase IV completion, where forelimb licking would have qualified as a Phase IV substitute. Although destriate/decorticate rats spend a large proportion of grooming time in forepaw licking, and do employ forepaw licking as a Phase IV substitute on occasion, they still generally fail to complete chains syntactically at normal rates even by this relaxed criterion. This demonstrates that the deficit is due to a failure to implement the syntactic rule, and not an inability to make the requisite movements.

\section{Microstructure of completed chains}

Videotaped syntactic chains from each group were examined closely in order to discover whether the fine structure of successful chains was altered by cortical or striatal damage (Fig. 5). The time from initiation to completion by the onset of Phase IV body licking is depicted in Fig. 6. Intact control rats reached Phase IV within $4.2 \pm 0.2 \mathrm{~s}$ from the emission of the first Phase I ellipse. Time from Phase I onset to Phase IV onset was not altered by any degree of cortical damage. Completion time was slightly prolonged in destriate/decorticate rats: rats lacking a striatum required an additional $1 \sec (24 \%$ increase) to reach Phase IV $(5.4 \pm 0.7 ; p<0.5$, ANOVA). The slight increase in chain duration for destriate/decorticate rats was probably due to a slight impairment of the speed of Phase III strokes, and not to an increase in the number of component strokes (Fig. 7). The number of forelimb strokes included in syntactic chains averaged $25 \pm 3$ strokes when Phases I, II, and III were combined (bilateral strokes made simultaneously with two forepaws were counted as two strokes). The number of strokes contained within a chain did not vary significantly between groups.

\section{Ellipse timing: phase I strokes}

Cortical and striatal lesions had no effect upon the rate $(\overline{\mathrm{x}}=6.2 \mathrm{~Hz})$ or duration $(\overline{\mathrm{x}}=160 \mathrm{~ms})$ of the elliptical trajectories of Phase I strokes $(F(3,21)=1.14, p>0.1)$. Two factors had a powerful influence upon the speed of the ellipse cycle, however, independent of lesion group (Fig. 8). First, Phase I ellipse speed was a useful predictor of whether the chain would be completed syntactically $(F(2,21)=25.2, p<0.001)$, as has been reported for intact rats and mice before (Berridge 1990). Ellipses were emitted at a faster rate at the beginning of chains that were

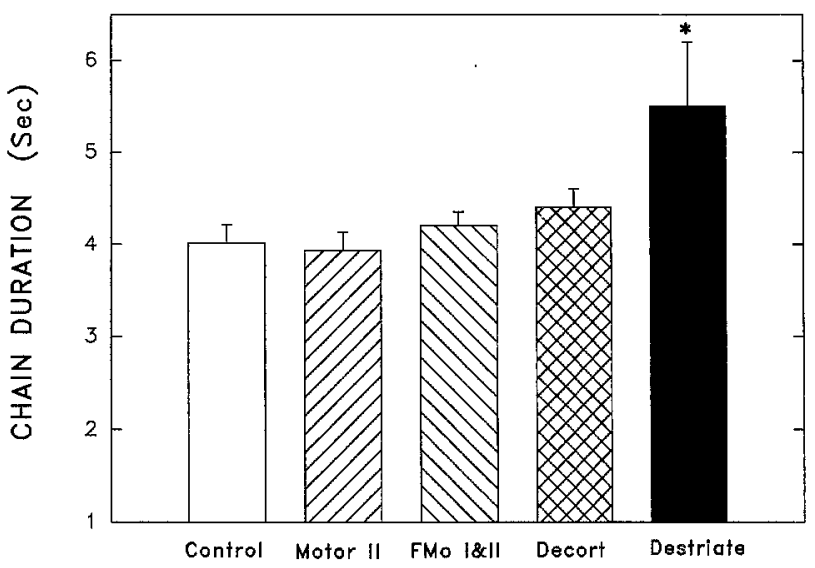

Fig. 6. Experiment 1. Duration of syntactic grooming chain. Bars show the mean $( \pm$ SEM) duration of completed chains for each group. Completion time was the time elapsed from the onset of Phase I (first ellipse) to the onset of Phase IV (body licking). Star denotes significant difference from all other groups $(p<0.05)$

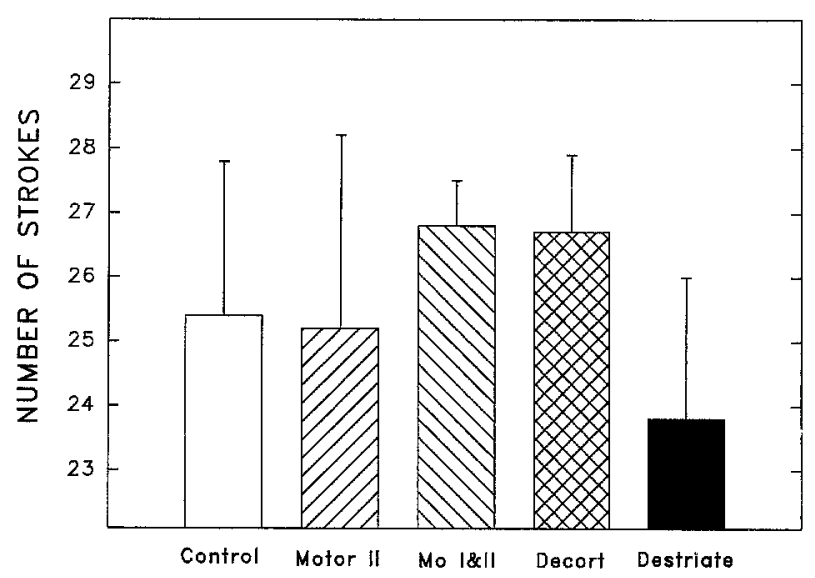

Fig. 7. Experiment 1. Total number forelimb strokes contained within syntactic chain. Strokes were counted as the number of 'peaks' shown in chain notations within Phases I, II, and III. Strokes were counted separately for each forepaw

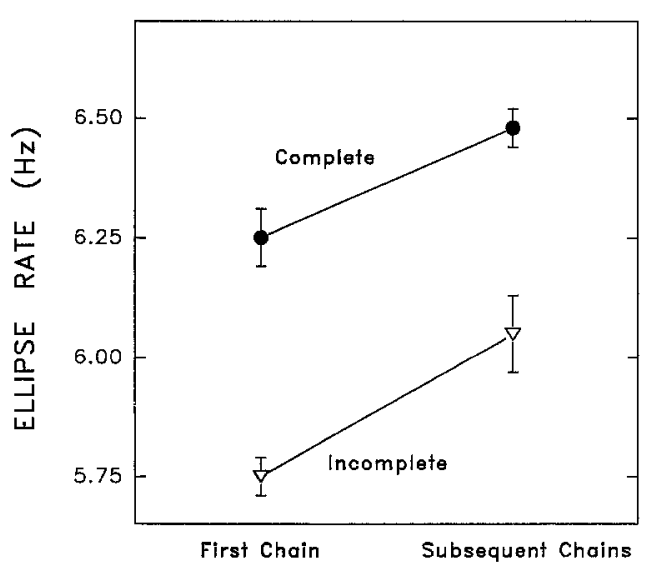

Fig. 8. Experiment 1. Speed of elliptical strokes. During the Phase I of chains that were subsequently completed through Phase IV, ellipses were emitted at a faster rate than equivalent ellipses of chains not completed syntactically. Independently of syntactic completion, ellipses were emitted at a slower rate during the first chain of a grooming session than during subsequent chains 
completed syntactically $(6.4 \mathrm{~Hz})$ compared to chains that failed to progress directly to Phase IV $(5.9 \mathrm{~Hz}$; Fig. 8). Second, the order of a chain within a session also controlled ellipse speed: another apparent "warm up" or behavioral inertia effect. The initial chain of any session was begun by slower ellipses than were later chains of the same session, regardless of whether or not the chain was completed syntactically (Fig. $8 ; F(1,21)=11.16$, $p<0.01$ ).

\section{Discussion}

Cortical lesions did not disrupt the completion of syntactic grooming chains nearly as dramatically as adding striatal ablation. Even total decortication produced only a very mild impairment of syntactic completion, compared to the deficit that followed removal of the striatum. Only early chains emitted at the beginning of a session within the test chamber were impaired by cortical lesions as long as the striatum was intact. Rats with cortical lesions were able to complete chains with near normal proficiency by the second chain of a session, and even total decorticates were nearly normal in completion rates for their third chain of a session. The magnitude of the "first-chain" impairment was roughly proportional to the size of the cortical lesion: full decorticates completed fewer "first chains" than rats whose lesions were restricted to motor cortex.

The additional loss of the striatum dramatically reduced the likelihood that a chain would be completed syntactically regardless of whether it was emitted early or late in a session. After loss of the striatum rats completed syntactic chains at only half the rate of normal rats, and never returned to normal levels. This deficit was one of sequencing rather than of a motor inability to emit the appropriate actions. Unlike the grooming interruptions caused by some brain lesions, such as "grooming arrest" after lateral hypothalamic damage in which rats lapse into somnolence while grooming (Levitt and Teitelbaum 1975), grooming did not usually cease or become displaced by another type of behavior when syntactic chains were left incomplete. Instead, grooming typically continued after a pause of $1-5 \mathrm{~s}$ but the serial order of grooming actions no longer followed the syntactic chain pattern.

The magnitude of the completion deficit shown by destriate/decorticate rats in this study was similar to the dificit that has been found for rats that have only nigrostriatal damage without cortical damage (Berridge 1989b), and similar also to the deficit that has been found for mesencephalic and metencephalic decerebrate rats, which possess only a midbrain (mesencephalic only), medulla oblongata, pons, and cerebellum (Berridge 1989a). By comparison, myelencephalic or "medullary" decerebrates in that earlier study, which were transected behind the pons, virtually never produced a perfect syntactic chain.

\section{Experiment 2}

Taken as a group, these studies might indicate that the generation of the syntactic pattern depends crucially upon an intact pons, and that implementation of the generated pattern at normal levels of accuracy requires an intact striatum (regardless of whether the cortex is intact). A reservation concerning this conclusion arises, however, from a procedural feature of the earlier decerebrate experiment: transections in that study which disconnected the pons from the medulla also produced substantial damage to the anterior cerebellum and cerebellar peduncles (Berridge 1989a). Cerebellar lesions have been shown to disrupt the sequential organization of trained responses and to impair the performance of "ballistic" actions (Buchtel et al. 1926; Pellegrino and Altman 1979; Thompson, 1974). Was it the loss of the brainstem pons or of the cerebellum that left medullary decerebrates unable to perform syntactic chains? It would be helpful to know whether cerebellar lesions by themselves disrupt the performance of syntactic chains during grooming.

\section{Method}

\section{Surgery}

Eight male and female Sprague-Dawley rats $(350-500 \mathrm{~g})$ were anesthetized with $100 \mathrm{mg} / \mathrm{kg}$ ketamine $\mathrm{HCl}$ and $10 \mathrm{mg} / \mathrm{kg}$ xylazine. The posterior skull was exposed, and a ribbon of bone $3 \mathrm{~mm}$ in width, from $-1 \mathrm{~mm}$ to $-3 \mathrm{~mm}$ posterior to lambda, and extending bilaterally between the left and right squamosal bones, was removed using rongeurs. The meninges were cut and retracted to expose the cerebellar vermis. The medial vermis, and the anterior, simple, and ansiform lobules were removed on both sides by aspiration. Bleeding was controlled with sterile gel-foam, and the wound was sutured. Bicillin ( $30000 \mathrm{U}$, im) was given, and repeated prophylactically every alternate day for 8 days after surgery. Five additional rats were used as surgical controls. For control rats, the ribbon of bone was removed just as for cerebellar aspiration, but the meninges were not damaged and the wound was sutured.

\section{Maintenance}

Rats were housed as in Experiment 1. Hypophagic rats were provided with a palatable wet cereal mash (Gerber's Mixed Baby's Cereal and water) fresh daily, in addition to chow pellets and water.

\section{Grooming}

Grooming was elicited and videotaped and analyzed for bouts and syntactic chains as in Experiment 1.

\section{Results}

Histology

Gross and histological inspection confirmed the elimination of most cerebellar tissue after aspiration lesions (Fig. 9). For all rats, ansiform and simple lobules were 

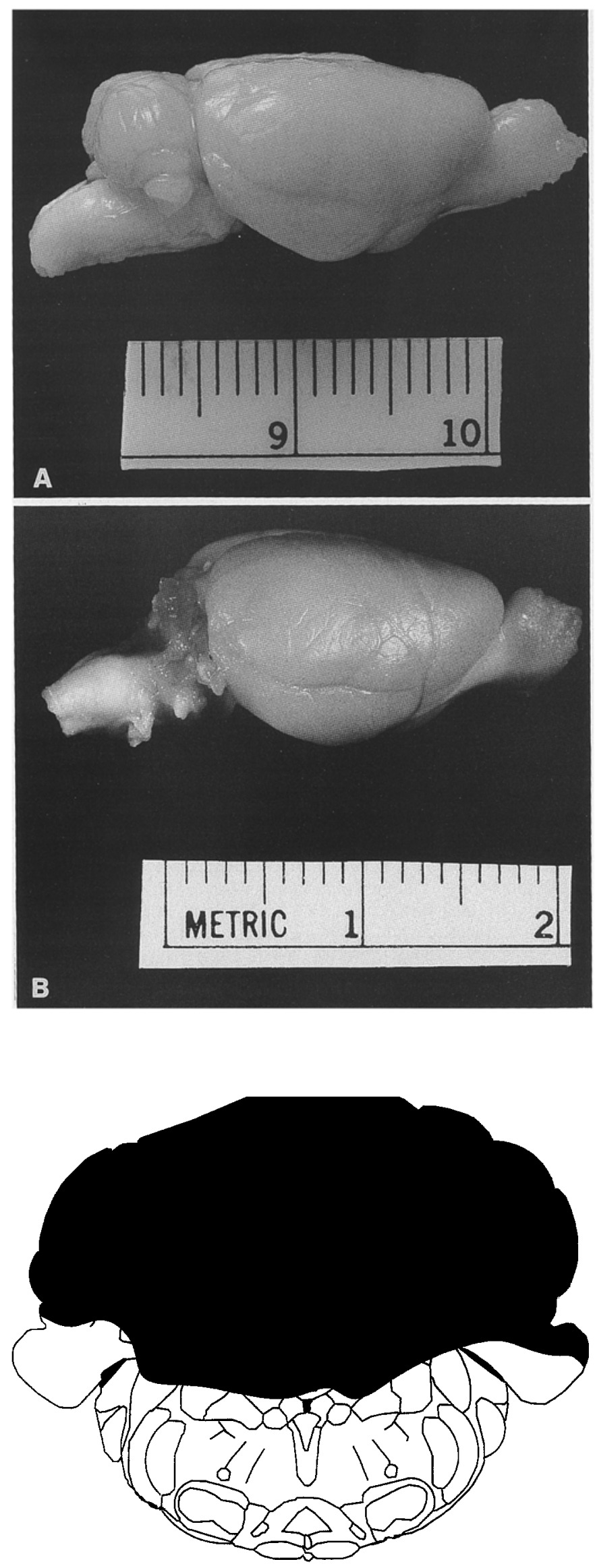

Bregma $-10.80 \mathrm{~mm}$ lacking on both sides, and medial lobules $3,4,5,6,7$, and 8 were removed. A small portion of the anterior lobe was preserved in all rats. Ventromedial lobes 1,2 , and 10 were preserved in four rats, and deep cerebellar nuclei were preserved in five rats.

\section{General condition and grooming}

Cerebellar lesions produced pronounced postural and locomotion deficits that recovered gradually over 3-10 days. Despite these deficits, all rats ate moistened cereal and maintained or increased body weight. During this early period, rats would still groom and emit syntactic chains if they were given external postural support and propped up by hand (much like medullary decerebrates [Berridge 1989a]). For this analysis, however, only data were used that were collected after postural recovery (after day 10), when rats groomed spontaneously on their own. Rats that had cerebellar damage emitted grooming bouts that were twice as long in duration as those of control rats (control $=19+7 \mathrm{~s}$, cerebellar-X $=40 \pm 13$; $F(1,11)=37.9, p<0.01)$.

\section{Syntactic chain completion}

Although cerebellar rats spent more time grooming than control rats, the rate of initiation of syntactic chains per minute of grooming did not differ between groups. Cerebellar ablation mildly impaired the syntactic completion of grooming chains $(F(1,11)=10.9, p<0.01)$ in a way that was similar to the effect of cortical lesions in Experiment 1 (Fig. 10). After ablation of the cerebellum, rats remained capable of generating good syntactic chains and most chains were completed syntactically to Phase IV.

The same "warm up" pattern of improved syntactic completion over the first, second, and third chains of a session, as described for Experiment 1, was seen for control rats and rats with cerebellar lesions $(F(2,22)=64.18, p<0.01)$. Cerebellar lesions magnified the normal first-chain deficit in syntactic completion, just as had cortical lesions (Fig. 10). Intact control rats successfully completed to Phase IV $79 \%$ of their initial chains within a grooming sessions, and rose to $98 \%$ complete for later chains of the same sessions. Rats with cerebellar lesions did not differ from control rats in completion rates for " $n+1$ " chains after the initial one (97\%), but were less likely than control rats to complete that first one successfully $(61 \% ; F(2,22)=6.04, p<0.01)$.

Fig. 9A, B. Experiment 2. Cerebellar lesions. A Photograph shows representative brains: control (top) and after cerebellar ablation (bottom). B Drawing shows the extent of damage that was common within the group 

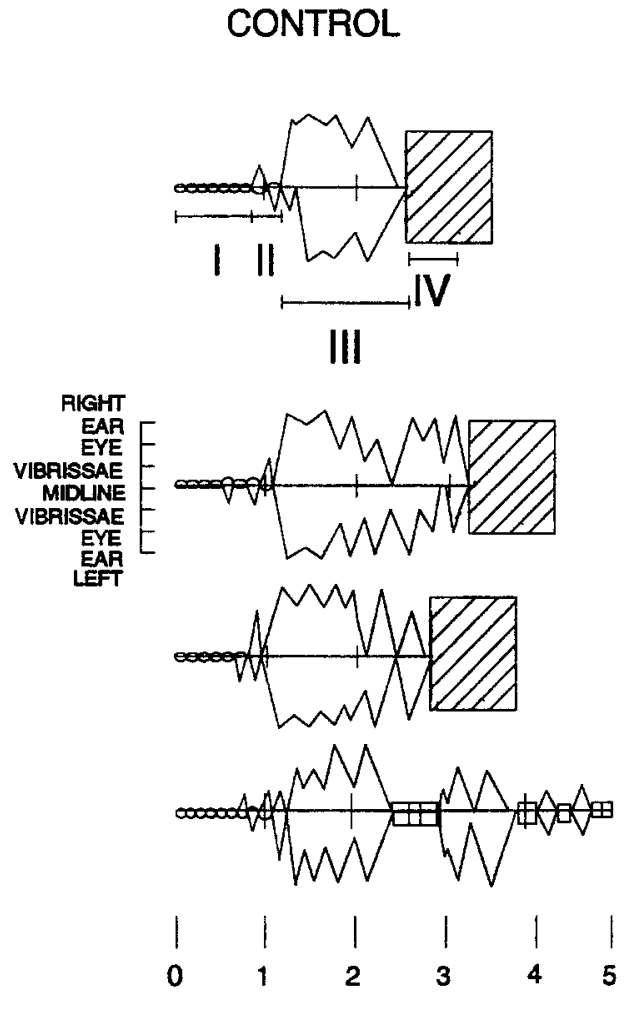

\section{CEREBELLAR ABLATION}
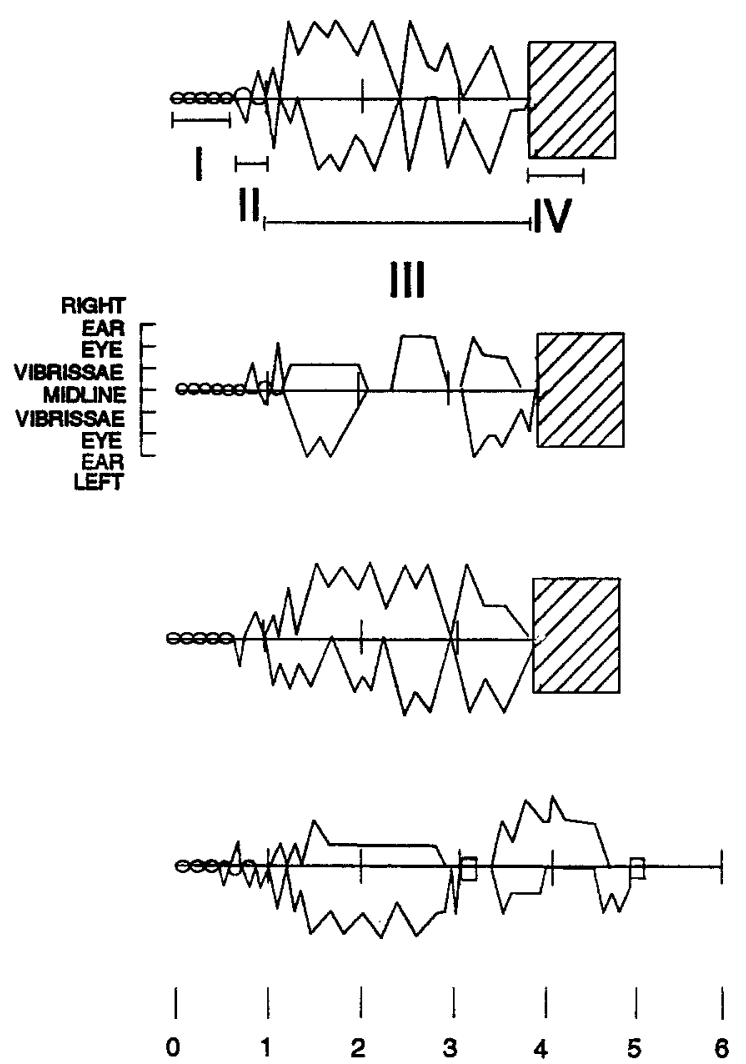

Fig. 10. Experiment 2. Notated syntactic chains after cerebellar ablation. Actual samples are shown of three syntactically complete chains and of one incomplete chain emitted by cerebellar and control groups. The occurrence of each phase is marked in the first top chain for each group. Time (in seconds) is shown at bottom. Symbols are as in Fig. 1 and 5

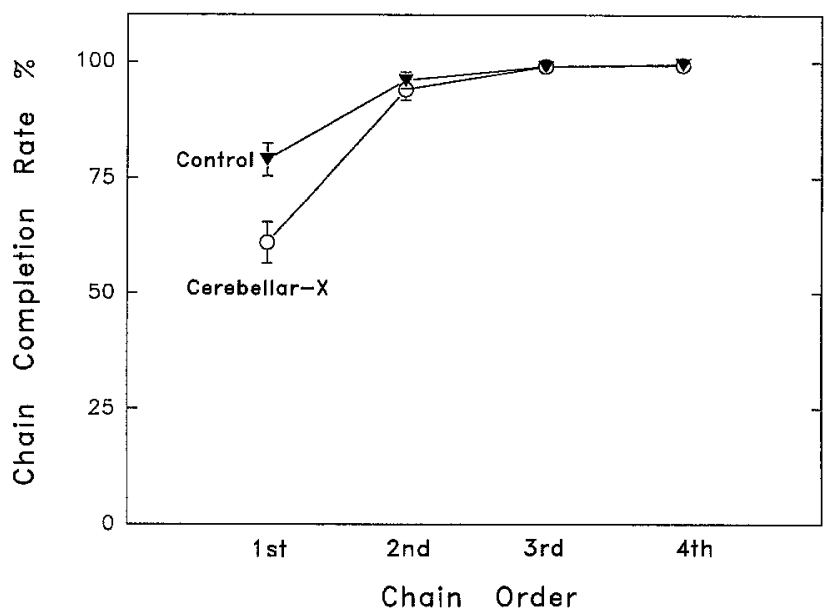

Fig. 11. Experiment 2. Syntactic chain completion: inertia effect is magnified by cerebellar ablation. The percentage of first, second, third, and forth grooming chains begun within a session that were completed syntactically (using the stringent criterion for completion). Cerebellar ablation potentiated the normal "first-chain completion deficit" in a manner similar to neocortex lesions

\section{Microstructure of completed chains}

The duration of syntactically complete chains, from the beginning of the first Phase I elliptical stroke to the onset of Phase IV body licking, did not differ significantly between control and cerebellum lesion groups (control $=3.1 \pm 0.3$, cerebellum $=3.4 \pm 0.5 ; F(1,11)=0.29$, $p>0.1$ ). However, 3 extra strokes were contained on average in the syntactic chains of cerebellum-lesion rats compared to the chains of control rats (cerebellum lesion $=27.3 \pm 2.1 \quad$ strokes $\quad$ vs. $\quad$ control $=24.4 \pm 1.7$ strokes; $F(1,11)=6.18, p<0.05)$. This increase in the number of strokes within the chain did not appear to arise from any single phase, but instead was distributed throughout the chain. An analysis of stroke number phase-by-phase found no difference in the number of Phase I ellipses or asymmetrical Phase II strokes between groups. The slightly greater number of bilateral Phase III strokes found in chains emitted by rats with cerebellar lesions $(19.7 \pm 1.7)$ compared to control chains did not quite reach significance $(18.0 \pm 1.1 ; \quad F(1,11)=4.07$, $p=0.067$ ).

\section{Ellipse timing}

Cerebellar lesions had no effect on the rate or duration of Phase I elliptical strokes, $(F(1,11)=0.31, p>0.1)$. Therefore timing data from cerebellar and control groups were combined for this analysis. Ellipse speed again proved to be a predictor of whether the chain would be completed syntactically to Phase IV $(F(1,12)=51.37, p<0.001)$, as in Experiment 1. Chains that had faster Phase I ellipses were more likely to be completed syntactically (Fig. 11). Chain order was also a significant factor controlling the rate of ellipse emission $(F(1,12)=7.50, p<0.02)$, again as in Experiment 1. The initial chain of a grooming session tended to have slower 


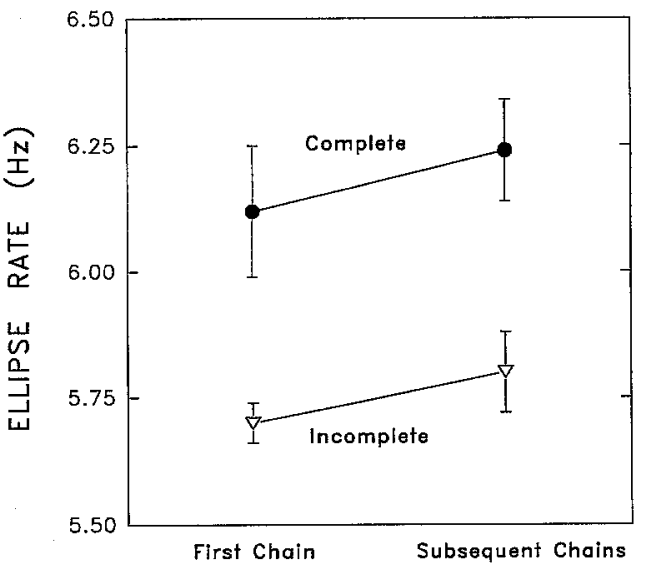

Fig. 12. Experiment 2. Speed of elliptical strokes in cerebellar and control rats (combined). Replication of the independent effects on rate of ellipse emission of chain completion and of chain order within a grooming session

Phase I ellipses, regardless of whether or not it was completed syntactically (Fig. 11).

\section{Discussion}

Removal of the cerebellar cortex increased the overall tendency to engage in grooming and did not seriously disrupt the sequential pattern of syntactic grooming chains. Only a mild deficit in chain completion was produced, which was very similar to the deficit produced by removal of the neocortex. This deficit was manifest as an exaggeration of the normal "behavioral inertia" effect on sequencing: the tendency not to complete the first chain of any grooming bout. After cerebellar or neocortical damage, rats were even less likely than normal to complete the first chain they began. Later chains from the same bout, however, were completed at normal levels.

The fragmentation of chain order that is found when one moves from a metencephalic decerebrate, which has a pons and cerebellum, to a myelencephalic "medullary" decerebrate, which lacks a pons and has a damaged cerebellum (Berridge 1989a), was not produced here by damage to the cerebellum alone. It would appear from these results that it is the loss of the pons that disrupts sequencing in the medullary decerebrate (compared to the pontine decerebrate), and not loss of the cerebellum. As far as the sequencing of syntactic grooming chains is concerned, the cerebellum appears not to specify the order or timing component actions but rather to magnify the relative deficit in coordination that occurs early in a grooming session. This is manifest as a enhanced "firstchain deficit" after cerebellar lesions.

\section{General discussion}

These experiments demonstrate that the striatum has a special role in the control of the serial order of grooming behavior. Lesions of the cortex or cerebellum merely amplified the early lag in competence that is revealed in the normal tendency to fail to complete the first chains of a grooming session. Exaggeration of a "warm up" period needed for motor competence has also been reported in rats after lesions of the lateral hypothalamus (Golani et al. 1979, and it is possible that these similar deficits are related.

By contrast, a permanent deficit in the syntactic completion of grooming chains appeared in this study only after the striatum was removed. This permanent striatal deficit was similar to the deficits in grooming syntax that have been found to occur after neurotoxin-induced damage to the striatum, and after brainstem transection at the pons or midbrain (Berridge and Fentress 1987b; Berridge 1989a, 1989b; Cromwell and Berridge 1990).

The unique importance of the striatum for grooming syntax is perhaps surprising for at least two reasons. First, because the striatum is massively connected to the neocortex by parallel loops of projections, and these corticostriatal circuits would have been equally affected by lesions of the motor cortex or striatum. Based upon current anatomical models of corticostriatal connections, one might have predicted that lesions of the striatum and of corresponding cortical areas would produce similar effects. Some behavior, such as skilled forelimb reaching, is disrupted by both cortical and striatal lesions in the rat (Whishaw et al. 1985). Yet cortex and striatal lesions did not produce similar consequences for behavioral sequencing in this study. A second reason why it may be surprising that cortical or cerebellar lesions failed to impair syntactic sequencing is that deficits in sequential performance on a variety of behavioral tasks have been reported to result from damage to either the frontal cortex or cerebellum. Each of these reasons is discussed below.

\section{Anatomical loops between cortex and striatum}

The dorsolateral region of the anterior neostriatum contains the striatal neurons crucial to the implementation of syntactic grooming chains. A recent study of the effects of small, localized excitotoxin lesions has shown that bilateral lesions only of this anterolateral region of the striatum are effective in disrupting the completion of syntactic chains (Cromwell and Berridge, 1990). Lesions of other striatal regions did not disrupt syntactic completion. A recent electrophysiological study has found that neurons within the same dorsolateral region appear to respond differently during the production of a syntactic grooming chain than during grooming outside the chain (Aldridge et al. 1990). The dorsolateral striatum receives extensive projections from the motor area of the rat neocortex (Kelley et al. 1982; Nauta and Domesick 1990 ) and is considered part of a major motor circuit for forelimb control (West et al. 1990; Pisa and Cyr 1990). Since an anatomical loop is formed by fibers passing from the motor cortex $\rightarrow$ dorsolateral striatum $\rightarrow$ ventral thalamus $\rightarrow$ premotor cortex $\rightarrow$ back to motor cortex (e.g., Nauta 1989), this cortex-striatum-cortex loop would have been disrupted equally by our lesions of the motor cortex, premotor cortex, and striatum. If this anatomical 
loop had operated as a single functional circuit to promote grooming sequences, then any of those lesions should have "short-circuited" the sequencing function (Divac et al. 1987). But instead the behavioral effects of these lesions were not equivalent. The fact that only striatal lesions impaired grooming syntax suggests that it is intrinsic processing within the striatum, instead of extrinsic connection to a cortical loop, that is most crucial for behavioral performance of this sequential pattern. These intrinsic striatal circuits must interact with projections from the brainstem structures that generate the pattern for syntactic chains (e.g., Manetto and Lidsky 1987), of course, but they do not require cortical assistance (e.g., Aldridge et al. 1990).

As a caveat to this conclusion, it must be admitted that our decortications did not destroy all paleocortex ventral to the rhinal fissure, and one might use this admission to argue that a corticostriatal loop crucial to syntactic grooming was spared by our cortical lesions. This seems implausible to us, however, because only lesions of the dorsolateral sector of the neostriatum disrupt syntactic chains (Cromwell and Berridge 1990). That striatal sector receives most of its cortical projections from the primary and supplementary motor neocortex region that was removed by our lesions, and from somatosensory neocortex that was eliminated by decortication, rather than from the paleocortex ventral to the rhinal fissure that was spared (Kelley et al. 1982; Nauta 1989). We conclude that corticostriatal loops are not the substrate for syntactic chain implementation.

\section{Encephalization of grooming: species differences}

There have been reports that both frontal cortex lesions and decortication can impair the sequencing of grooming by rats, in the sense that grooming bouts after such lesions were shortened and were interrupted by other behavior such as walking (Kolb et al. 1977; Vanderwolf et al. 1978). However, further studies have shown that this cortical "sequencing deficit" is context-dependent, and not really due to an inability to sequence. Decorticate rats showed the shorter bouts only when tested in a novel environment, and not when they were grooming either in their home cage or in an environment with which they were thoroughly familiar (Whishaw et al. 1981; Whishaw 1990). In the present study, the rats became familiar with the test environment, and no longer showed this context-specific deficit after the 10th day of testing. Taken together, these studies confirm that the rat neocortex is not needed for the sequential patterns of grooming, but may be involved in more general functions such as habituation and alertness to environmental cues, the tendency to explore, etc., that may influence the performance of grooming.

In primates, by contrast, decortication and focal cortex lesions can seriously disrupt grooming performance. Self-grooming by monkeys, and washing and dressing by humans (as well as most other behavior) may vanish completely after severe cortical damage (e.g., Kolb and
Milner 1981). What is the nature of this difference in cortical function between rodents and primates?

It would be rash to suppose that the locus of grooming control has moved from the striatum to the cortex in the course of primate evolution. It might be more accurate to say that control has spread to include the cortex as well as the striatum. The crucial difference is that in primates, unlike in rats, the striatal coordination of grooming incorporates cortical circuitry to a degree that the function can no longer be carried out when the cortex is suddenly lost. Although corticostriatal loops are present anatomically in both rodents and primates, they possess a function importance in primates that is not shared in rodents. Grooming by primates is less stereotyped, more influenced by learning, more highly social, and uses more independent limb movements than grooming by rats. These aspects of primate grooming may well require a greater contribution from neocortex. The effects of cortical lesions in primates thus extend to grooming behavior, whereas the analogous behavior is more robustly subcortical in other mammals.

\section{Sequencing functions of frontal cortex and cerebellum}

Lesions of the frontal cortex or cerebellum both have been shown to disrupt many sequential aspects of behavior in a variety of species including rats. Frontal cortex lesions, for example, disrupt the ability of human patients to generate sequences of novel responses in tasks where the chief goal is to copy a series of movements (Kolb and Milner 1981) or to ensure that no response repeat an earlier one (Petrides and Milner 1982). Humans, monkeys and rats all tend inappropriately to repeat previously rewarded or dominant responses after damage to the frontal cortex on a variety of learning tasks where success requires switching between responses (Goldman-Rakic 1987; Iversen and Mishkin 1970; Kolb 1984). Monkeys with damage restricted to the supplementary motor cortex can perform movements that are initiated by external signals but are impaired when the learned response sequence must be internally retrieved and initiated, as if the internal "switching on" of the pattern was disrupted (Passingham et al. 1989).

Damage to the cerebellum also impairs sequential performance on certain behavioral tasks. Cerebellar lesions disrupt the completion of fast "ballistic" actions, such as saccadic eye movements, in which the pattern of movement must be internally programmed in advance of execution (Dichgans 1984). Cerebellar lesions also produce perseverative deficits in learning tasks, such as repetition of the same response by rats in operant chambers or in mazes where successful performance requires, for example, alternating turns to the left and right (Buchtel 1970; Kirk et al. 1982; Lashley and McCarthy 1926; Pellegrino and Altman 1979; Thompson 1974).

Why do cerebellar and cortex lesions disrupt the sequencing of behavior by rats in such tasks yet not in syntactic grooming? The answer must be that the sequencing of those types of behavior is mediated by functions different from the ones studied here. Ballistic 
reaching or eye movements require sequential programming for a single, rapid action. The performance of a memory-based series of instrumental responses in a maze or sorting task depends upon cognitive representations acquired through learning. Behavior such as learned instrumental alternation or ballistic movements may well require functions mediated by circuits in the cortex and cerebellum. Frontal cortex, for example, has been argued to be needed for the sustained activation of a learned representation of an event or relation, which would be required in order to successfully perform trained sequences (Goldman-Rakic 1987). The organization of instinctive actions into natural rule-governed sequences, on the other hand, depends upon very different functions. Those functions are, first, to generate sequential action patterns that contain multiple actions according to a rule and, second, to impose the patterns upon the stream of motor output. Those are the functions crucial to the syntactic completion of grooming chains, and those functions appear to be mediated chiefly by the striatum and brainstem.

\section{Role of the striatum in syntactic grooming}

Despite the sequential grooming deficit that follows striatal lesions, the nature of the striatal contribution need not be either to generate component grooming actions or to specify the serial order of the syntactic chain pattern (MacLean 1990). Evidence indicates that generation both of the movements themselves and of the sequential pattern studied here is accomplished primarily within the brainstem (Berntson et al. 1988; Berridge 1989a). An hypothesis for the role of the striatum might be instead that it implements the pattern by modulating the activation of competing sensorimotor and central pattern generating circuits elsewhere in the brain (Iversen 1979; Van den Bercken and Cools 1982; Cools 1985). The striatum may promote the completion of a syntactic grooming chain through a phasic and selective facilitation of the access of the chain's brainstem syntax generator to motor output systems, while simultaneously channeling motor control access away (probably in a graded fashion) from competing programs and sensorimotor circuits. Support for this "phasic switch" hypothesis comes from other studies, which have indicated that a phasic suppression of sensorimotor responsiveness occurs during the performance of a syntactic grooming chain (Berridge and Fentress 1986; unpublished observations). The striatum may mediate this phasic switching of movement control between sensory-guided systems and centrally-generated syntactic programs. If so, then its role is to act as a hierarchic controller which turns on and off other circuits in the brainstem and elsewhere as behavior progresses (Gallistel 1980).

\section{Implications and conclusions}

The hypothesis that the striatum controls the serial order of grooming via hierarchical modulation may have im- plications for certain human clinical disorders. The hypothesis can be restated in a more general form, name$1 y$, that the striatum modulates the serial activation of brain circuits that represent discrete functions, both those that mediate thought, as well as those that mediate action. This general hypothesis can accommodate suggestions that the striatum is involved in the pathological perseverations of action and thought seen in human Tourette's syndrome, obsessive-compulsive disorder, etc., which have been made by Rapoport and others (Cummings and Frankel 1985; Rapoport and Wise 1988; Rapoport 1989). The essential nature of the hierarchical modulating function need not have changed from the one that mediates grooming syntax in order to apply also to perseverative sequences of thought. Instead, it is only necessary to suppose that the class of elements that is modulated hierarchically by the striatum has been expanded during human evolution to extend beyond mere action functions (sensorimotor circuits, pattern generators) to cognitive functions too (abstract features and symbols).

Is it plausible to argue that the striatum modulates cognitive, as well as motor, aspects of human action? Evidence can be found to support the argument that the human striatum is especially important to cognitive aspects of movement. For example, when asked to use common tools (hammer, comb, scissors) in an appropriate way, a substantial percentage of patients with Huntington's disease made conceptual - not motor errors, either confusing the tool with their own hand and making movements without the tool, or confusing the tool they were asked to use with a different one (Shelton and Knopman 1991). Other investigators have reported correlations between striatal pathology and sequencerelated cognitive deficits in monkeys (e.g., Schneider and Kovelowski 1990) and between striatal abnormality and perseverative language and thought disorders in humans (Brunner et al. 1982; Luxenberg et al. 1988). If modulation by the striatum has been extended by human evolution to circuits that mediate representations of events, objects, and symbols, in addition to actions, then sequential patterns of thought and language could be controlled in the same way as sequential patterns of grooming (Marsden 1982). Excessive facilitation of particular patterns of cognitive elements might well produce the stereotyped thought and habits of obsession or verbal repetition of Tourette's syndrome, just as the stereotyped syntax of grooming chains is strengthened by striatal modulation.

In conclusion, behavioral sequencing depends upon many separable brain functions. Different functions are involved to varying degrees in the sequencing of different types of behavior, and these functions are mediated by separate brain systems. Some functions may apply to all behavioral sequences; other functions to only certain types. Selection of a particular type of behavior for study determines the relative importance of a particular sequencing funtion. Learned sequential performance has been shown by others to be disrupted by lesions of many neural structures. Instinctive behavioral sequences by comparison might be said to depend only upon "mere 
action syntax': the capacity to generate rules of serial order, and to activate those rules and impose them upon behavioral actions. Our results demonstrate a preeminent role for the striatum in the control of action syntax. Only lesions of the striatum disrupted the completion of syntactic chains of grooming actions permanently. Lesions of the neocortex or cerebellum, by comparison, appeared to affect only a minor and phasic factor that was represented as an increase in "warm up" deficits. We suggest that the special role of the striatum in facilitating the performance of syntactic chains involves a phasic hierarchical channelling of action control between centrally-generated patterns and sensory-guided circuits. Finally, an evolutionary extension of the same striatal function that facilitates syntactic grooming chains in rats might also modulate human sequences of learned action, language, and thought. Distortion of this striatal function by striatal-related disease may be responsible for some of the stereotypies of action, speech, and thought that characterize human Tourettes syndrome, obsessive-compulsive disorder, and other related disorders.

Acknowledgements. We are grateful to Dr. J. Wayne Aldridge and Howard C. Cromwell for helpful comments on an earlier manuscript of this article. We also thank John Kim, Darlene Tansil, and a number of University of Michigan undergraduates for help with animal care and with the behavioral testing and analysis. We thank Dr. J. Wayne Aldridge for the drawing of Figure 1. Care for animals conformed fully with NIH, NSERC, and university (Lethbridge and Michigan) guidelines. This study was supported by grants from the NIH (NS 23959 to K.B.) and the NSERC (to I.W.)

\section{References}

Aldridge JW, Berridge KC, Herman M (1990) Single unit activity in the rat caudate-putamen during stereotypical grooming sequences. Neurosci Abstr 16:233

Aldridge JW, Gilman J, Dauth G (1990) Spontaneous neuronal unit activity in the primate basal ganglia and the effects of precentral cerebral cortical ablations. Brain Res 516:46-56

Barone P, Joseph JP (1989) Prefrontal cortex and spatial sequencing in macaque monkey. Exp Brain Res 78:447-464

Berntson GG, Jang JF, Ronca AE (1988) Brainstem systems and grooming behaviors. Ann New York Acad Sci $525: 350-362$

Berridge KC (1989a) Progressive degradation of serial grooming chains by descending decerebration. Behav Brain Res $33: 241-253$

Berridge KC (1989b) Substantia nigra 6-OHDA lesions mimic striatopallidal disruption of syntactic grooming chains: a neural systems analysis of sequence control. Psychobiology $17: 377-385$

Berridge KC (1990) Comparative fine structure of action: rules of form and sequence in the grooming patterns of six rodent species. Behavior 113:1-2

Berridge KC, Fentress JC (1986) Contextual contról of trigeminal sensorimotor function. J Neurosci 9:325-330

Berridge KC, Fentress JC (1987a) Deafferentation does not disrupt natural rules of action syntax. Behav Brain Res 23:69-76

Berridge KC, Fentress JC (1987b) Disruption of natural grooming chains after striatopallidal lesions. Psychobiology 15:336-342

Brunner RS, Kornhuber HH, Seemüller E, Suger G, Wallesch C-W (1982) Basal ganglia participation in language pathology. Brain Language 16:281-299

Buchtel HA (1970) Visual-learning deficits following cerebellar damage in rats. J Comp Physiol Psychol 72:296-305
Cools, AR (1985) Brain and behavior: hierarchy of feedback systems and control of input. In: Bateson PPG, Klopfer PH (eds.) Perspectives in ethology. Plenum, New York, 109-168

Cromwell HC, Berridge KC (1990) Anterior lesions of the corpus striatum produce a disruption of stereotyped grooming sequences in the rat. Neurosci Abstr 16:233

Cummings JL, Frankel M (1985) Gilles de la Tourette syndrome and the neurological basis of obsessions and compulsions. Biol Psychiatr 20:1117-1126

Dichgans J (1984) Clinical symptoms of cerebellar dysfunction and their topodiagnostic significance. Human Neurobiol 2:269-279

Divac I, Oberg GE, Rosenkilde CE (1987) Patterned neural activity: implications for neurology and neuropharmacology. In: Schneider JS, Lidsky TI (eds.) Basal ganglia and behavior: sensory aspects of motor fractioning. Huber, Toronto, pp 61-70

Donoghue JP, Wise SP (1982) The motor cortex of the rat: cytoarchitecture and microstimulation mapping. J Comp Neurol 212:76-88

Evarts EV, Wise SP (1984) Basal ganglia outputs and motor control. Ciba Found Sympos 107:83--102

Fentress JC (1983) Ethological models of hierarchy and patterning of species-specific behavior. In: Satinoff E, Teitelbaum P (eds.) Handbook of behavioral neurobiology. Plenum, New York, pp 185-234

Fentress JC, Stilwell F (1973) Grammar of a movement sequence in inbred mice. Nature (Lond) 244:52-53

Ferron J, Lefebvre L (1982) Comparative organization of grooming sequences in adult and young sciurid rodents. Behaviour $81: 110-127$

Gallistel CR (1980) The organization of action: a new synthesis. Erlbaum, Hillsdale NJ

Golani I, Wolgin DL, Teitelbaum P (1982) A proposed natural geometry of recovery from akinesia in the lateral hypothalamic rat, Brain Res 164:237-67

Goldman-Rakic PS (1987) Circuity of primate prefrontal cortex and regulation of behavior by representational memory. Hand Physiol 5:373-417

Iversen SD, Mishkin M (1979) Behavior after neostriatal lesions in animals. In: Divac I, Oberg RGE (eds) The neostriatum. Pergamon, New York, 183-195

Iversen, SD, Mishkin, M (1970) Perseverative interference in monkeys following selective lesions of the inferior prefrontal convexity. Exp Brain Res 11:376-386

Kelley AE, Domesick VB, Nauta WJH (1982) The amygdalostriatal projection in the rat. An anatomical study by anterograde and retrograde tracing methods. Neuroscience 615630

Kirk WT, Berntson GG, Hothersall D (1982) Effects of paleocerebellar lesions on DRL performance in the albino rat. J Comp Physiol Psychol 96:348-360

Kolb B (1984) Functions of the frontal cortex of the rat: A comparative review. Brain Res $8: 65-98$

Kolb B \& Milner B (1981) Performance of complex arm and facial movements after focal lesions. Neuropsychology 19:505-514

Kolb B, Whishaw IQ \& Schallert T (1972) Behavior sequencing, sensory neglect, and bodyweight set point following orbital frontal lesions in rats. Physiol Behav 19:93-102

Kurata K, Wise SP (1988) Premotor and supplementary motor cortex in Rhesus monkeys: neuronal activity during externally - and internally - instructed motor tasks. Exp Brain Res $72: 237-284$

Lashley KS (1951) The problem of serial order in behavior. In: Jeffress LA (ed) Cerebral mechanisms in behavior. Wiley, New York, pp 112-136

Lashley KS, McCarthy DA (1926) The survival of the maze habit after cerebellar lesions. J Comp Psychol 6:423-433

Levitt DR, Teitelbaum P (1975) Somnolence, akinesia, and sensory activation of motivated behavior in the lateral hypothalamic syndrome. Proc Nat Acad Sci 72:2819-2823

Luxenberg JS, Swedo SE, Flament MF, Friedland RP, Rapoport JL, Rapoport SI (1988) Neuroanatomical abnormalities in ob- 
sessive-compulsive disorder detected with quantitative $\mathrm{x}$-ray computed tomography. Am J Psychiatr 145:1089-1093

MacLean PD (1990) The Triune Brain in evolution: Role in Paleocerebral Functions. New York: Plenum

Manetto C, Lidsky TI (1987) Influences of the basal ganglia on the medullary reticular formation. Neurosci Let 75:278-282

Marsden CD (1982) The mysterious motor function of the basal ganglia: The Robert Wartenberg lecture. Neurology $32: 514-539$

Nauta WJH, Domesick VB (1984) Afferent and efferent relationships of the basal ganglia. In: Evered D, O'Connor $M$ (eds) Functions of the basal ganglia: Ciba Foundation Symposium. Pitman, London, 107:3-23

Neafsey EJ (1990) The complete ratunculus: output organization of layer V of the cerebral cortex. In Kolb B, Tees RC (eds) The cerebral cortex of the rat. MIT, Cambridge, pp 197-212

Neafsey EJ, Bold EL, Haas, G, Hurley-Gius KM, Quirk G, Sievert CF, Terreberry RR (1986) The organization of the rat motor cortex: a microstimulation mapping study. Brain Res Rev $11: 77-96$

Passingham RE, Chen YC, Thaler D (1989) Supplementary motor cortex and self-initiated movement. In: Ito M (ed) Neural programming. Karger, Basel, pp 13-24

Paxinos G, Watson G (1989) The rat brain in stereotoxic coordinates. Academic New York

Pellegrino LJ, Aitman J (1979) Effects of differential interference with postnatal cerebellar neurogenesis on motor performance, activity level, and maze learning of rats: a developmental study. J Comp Physiol Psychol 93:1-33

Pisa M, Cyr J (1990) Regionally selective roles of the rat's striatum in modality-specific discrimination learning and forelimb reaching. Behav Brain Res 37:281-292

Petrides M, Milner B (1982) Deficits on subject-ordered tasks after frontal- and temporal-lobe lesions in man. Neuropsychology $20: 249-262$

Rapoport JL (1989) The boy who couldn't stop washing: the experience and treatment of obsessive-compulsive disorder. Penguin Books, New York
Rapoport JL, Wise SP (1988) Obsessive-compulsive disorder: evidence for basal ganglia dysfunction. Psychopharm Bull $24: 380-384$

Richmond G, Sachs BD (1980) Grooming in Norway rats: the development and adult expression of a complex motor pattern. Behavior 75:82-96

Schneider JS, Kovelowski CJ 2d. (1990) Chronic exposure to low doses of MPTP. In: Cognitive deficits in motor asymptomatic monkeys. Brain Res 519, 122-128

Shelton PA, Knopman DS (1991) Ideomotor apraxia in Huntington's disease. Arch Neurol 48:35-41

Thompson R (1974) Localization of the "maze memory system" in the white rat. Physiol Psychol 2:1-17

Vanderwolf CH, Kolb B, Cooley RK (1978) Behavior of the rat after removal at the neocortex and hippocampal dormation. J Comp Physiol 92:156-175

Van den Bercken JH, Cools AR (1982) Evidence for a role of the caudate nucleus in the sequential organization of behavior. Behav Brain Res 4:319-327

West O, Carelli RM, Pomerantz M, Cohen SM, Gardner JP, Chapin JK, Woodward DJ (199) A region in the dorsolateral striatum of the rat exhibiting single-unit correlations with specific locomotor limb movements. J Neurophysiol 64:1233-1246

Whishaw IQ (1990) The decorticate rat. In: Kolb B, Tees RC (eds) The cerebral cortex of the rat. MIT, Cambridge, pp 239-268

Whishaw IQ, Kolb M, Sutherland RJ (1983) The analysis of behavior in the laboratory rat. In: Robinson TE (ed) Behavioral approaches to brain research. Oxford University Press, New York, pp 141-211

Whishaw IQ, O'Connor WT, Dunnett SB (1985) Disruption of central cholinergic systems in the rat by basal forebrain lesions or atropine: effects on feeding, sensorimotor behaviour, locomotor activity, and spatial navigation. Behav Brain Res 17: 103-115

Whishaw IQ, Nonneman AJ, Kolb B (1981) Environmental constraints on motor abilities used in grooming, swimming, and eating by decorlicate rats. J Comp Physiol Psychol 95:792-804

Zilles K (1990) Anatomy of the neocortex: cytoarchitecture and my eloarchitecture. In: Kolb B, Tees RC (eds) The cerebral cortex of the rat. MIT, Cambridge, pp 77-112 